\title{
Electropolymerized Poly(toluidine blue O) Film Electrode for Potentiometric Biosensing
}

\author{
Hiroto Satake and Toshiya Sakata* \\ Department of Materials Science and Engineering, School of Engineering, The University of Tokyo, \\ 7-3-1 Hongo, Bunkyo-ku, Tokyo 113-8656, Japan
}

(Received March 16, 2018; accepted April 27, 2018)

Keywords: toluidine blue O (TBO), electropolymerization, $\mathrm{pH}$ responsivity, potentiometric biosensor, sulfated glycosaminoglycan (sGAG)

In this study, we modified a poly(toluidine blue O) (TBO) film on a Au electrode by electropolymerization and investigated its electrical characteristics when used as a potentiometric biosensor. The fundamental properties of poly-TBO films such as immobilization density and thickness were clarified by atomic force microscopy (AFM) for different electropolymerization times. The poly-TBO film electrode with a spikelike surface, which was formed during electropolymerization, showed $\mathrm{pH}$ sensitivity $(45.5 \mathrm{mV} / \mathrm{pH})$ near the Nernstian response when used as a potentiometric sensor. This behavior is assumed to arise because the charge density at a spikelike film is higher than that at a smooth film. Thus, a platform based on a potentiometric biosensor with a poly-TBO film electrode can be used as a bioanalytical system to measure ionic behaviors that involve biomolecular recognition. This will enable the measurement of negatively charged sulfated glycosaminoglycan (sGAG), which is included in the extracellular matrix (ECM) generated from chondrocytes and is used as an important indicator in the development of articular cartilage in the field of regenerative medicine because of its electrostatic binding to positively charged TBO molecules.

\section{Introduction}

Chondrocytes have been studied in the field of regenerative medicine for the development of articular cartilage, which has a limited capacity for self-recovery owing to its avascular and anural nature. ${ }^{(1,2)}$ A regenerative medical technique to transplant cultured autologous chondrocytes has been developed, and many studies have been conducted to optimize the conditions of culture scaffolds ${ }^{(3-5)}$ and the effects of biochemical ${ }^{(6-12)}$ and physical stimulation $^{(13-16)}$ to improve the quality of cultured cartilage. Our group previously demonstrated the use of a semiconductor-based potentiometric biosensor with a chondrocytecoupled gate electrode for monitoring the interfacial $\mathrm{pH}$ between cultured chondrocytes and the gate electrode in real time following the addition of growth factors. ${ }^{(17)}$

*Corresponding author: e-mail: sakata@biofet.t.u-tokyo.ac.jp https://doi.org/10.18494/SAM.2018.1941 
To evaluate regenerative chondrocytes, the generation of an extracellular matrix (ECM) of, for example, collagen and proteoglycan is generally targeted. In particular, sulfated glycosaminoglycan (sGAG), which is included in proteoglycan, is typically quantified by staining tissues. ${ }^{(18)}$ In this case, toluidine blue O (TBO) is often utilized to stain the tissue because it binds electrostatically to the negatively charged sGAG. ${ }^{(18,19)}$ However, in the evaluation of stained tissues or cells, they are embedded in paraffin or destroyed in spectrometry. Such methods are invasive for tissues and cells and also time-consuming as experimental procedures. In addition, the information obtained is analyzed at the end point of biological phenomena and not measured in real time. Therefore, a novel method is required to noninvasively monitor sGAG in situ for the evaluation of living chondrocytes.

TBO acts as a receptor for sGAG on a sensor surface. The adsorption of negatively charged sGAG on positively charged TBO is based on electrostatic interactions. That is, a change in the molecular charge density of sGAG can be directly detected using TBO-modified potentiometric sensors. As a method of modifying TBO on electrodes, electropolymerization can be easily used for the direct polymerization of TBO on a conducting electrode [Fig. 1(a)]. ${ }^{(20)}$ Over the last few decades, various organic monomers have been coated on conducting substrates by electropolymerization. ${ }^{(21-27)}$

In this study, we modified a poly-TBO film on a $\mathrm{Au}$ electrode by electropolymerization and investigated its electrical characteristics when used as a potentiometric biosensor. The fundamental properties of poly-TBO films such as immobilization density and thickness were examined for different electropolymerization times. In addition, the performance of a polyTBO film electrode as a potentiometric sensor was evaluated on the basis of $\mathrm{pH}$ sensitivity. The knowledge obtained in this study should be applicable to the development of future sGAG sensors.

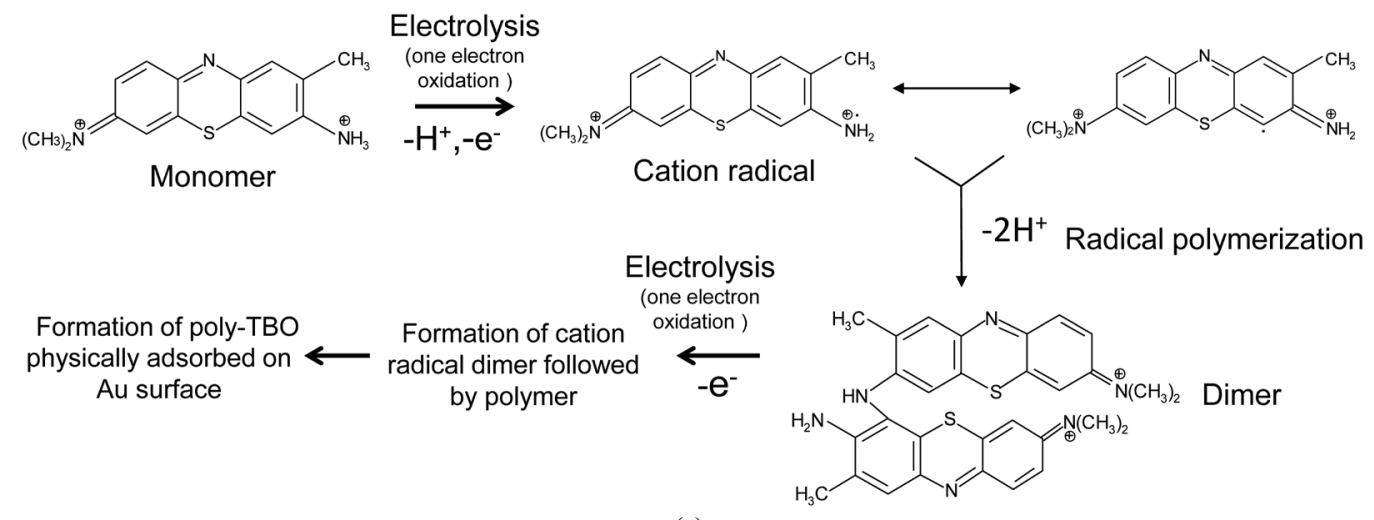

(a)
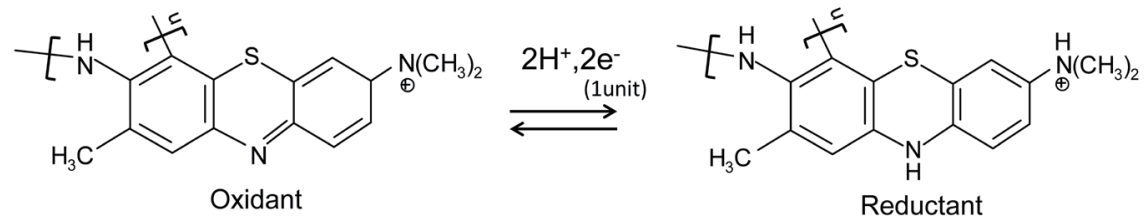

(b)

Fig. 1. (a) Electropolymerization of TBO. (b) Redox reaction of poly-TBO. 


\section{Experimental Methods}

\subsection{Preparation of poly-TBO-modified Au electrode}

A Au electrode was prepared by sputtering. First, Cr (thickness $15 \mathrm{~nm}$ ) was sputtered as an adhesion layer, after which $\mathrm{Au}$ (thickness $100 \mathrm{~nm}$ ) was sputtered. An 18-mm-diameter glass ring was attached to the Au substrate using epoxy resin. The Au electrodes were cleaned by piranha etching, then modified using TBO by electropolymerization in a solution containing 10 $\mathrm{mM} \mathrm{TBO}$ and $1 \mathrm{M} \mathrm{HCl}$ at a constant potential of $0.85 \mathrm{~V}$ [vs Ag/ $\mathrm{AgCl}$ (in saturated $\mathrm{KCl}$ )] for 1, 2, 4,6 , or $8 \mathrm{~min}$. The Au electrodes modified by poly-TBO were washed by soaking in deionized water overnight to remove nonspecifically adsorbed TBO molecules.

\subsection{Cyclic voltammetry measurement of poly-TBO-modified Au electrodes}

Cyclic voltammetry (CV) measurement was conducted on poly-TBO Au electrodes modified by electropolymerization to evaluate the redox peak and immobilization density of TBO. A poly-TBO-modified Au electrode was used as the working electrode. The reference and counter electrodes were a $\mathrm{Ag} / \mathrm{AgCl}$ electrode in saturated $\mathrm{KCl}$ and a platinum wire, respectively. The cyclic potential was swept from 0.7 to -0.2 or $-0.3 \mathrm{~V}$ (vs $\mathrm{Ag} / \mathrm{AgCl}$ in saturated $\mathrm{KCl}$ ) at $50 \mathrm{mV} / \mathrm{s}$ in $1 \mathrm{M} \mathrm{HCl}$.

\subsection{Evaluation of poly-TBO surface by atomic force microscopy (AFM)}

A drop of resist reagent (OFPR800, Tokyo Ohka Kogyo Co., Ltd.) was placed on a slide glass and heated on a hot plate $\left(120^{\circ} \mathrm{C}\right)$ to cure a circular region of the resist reagent. In the same way as in Sect. 2.1, a $\mathrm{Au} / \mathrm{Cr}$ thin film was coated on the slide glass with the cured resist by sputtering. Then, the $\mathrm{Au}$ electrode was soaked in acetone to remove the $\mathrm{Au} / \mathrm{Cr}$ film on the resist. Thus, regions both with and without the sputtered $\mathrm{Au} / \mathrm{Cr}$ thin film were formed on the slide glass, and the thickness of the $\mathrm{Au} / \mathrm{Cr}$ thin film on the slide glass plane was measured by AFM. Additionally, poly-TBO films were modified on the Au electrode by electropolymerization at a constant potential of $0.85 \mathrm{~V}(\mathrm{vs} \mathrm{Ag} / \mathrm{AgCl}$ reference electrode) in a solution containing $10 \mathrm{mM} \mathrm{TBO}$ and $1 \mathrm{M} \mathrm{HCl}$ for 1 or $6 \mathrm{~min}$. The poly-TBO-modified Au electrode was washed by soaking in deionized water overnight and then dried in a vacuum desiccator overnight. The total thickness of the $\mathrm{Au} / \mathrm{Cr}$ thin film and poly-TBO film was measured by AFM, and then the thickness of the poly-TBO film was evaluated as the difference between the total thickness and the thickness of the $\mathrm{Au} / \mathrm{Cr}$ thin film. The AFM measurement was carried out in the tapping mode.

\subsection{Measurement of $\mathrm{pH}$ responsivity of poly-TBO-modified Au electrode}

The surface potential of the poly-TBO film electropolymerized for 1 or 6 min was measured using an electrometer. In this case, $\mathrm{pH}$ responsivity was examined in a stepwise manner at $\mathrm{pH}$ 
values of 8.1, 6.9, 5.7, 4.7, 3.8, and 2.5. Each buffer solution was adjusted using $0.1 \mathrm{M}$ phosphate buffer to obtain $\mathrm{pH}$ values of 8.1, 6.9, and 5.7, and using $0.1 \mathrm{M}$ citrate buffer to obtain $\mathrm{pH}$ values of 4.7, 3.8, and 2.5. $\mathrm{A} \mathrm{Ag} / \mathrm{AgCl}$ electrode in saturated $\mathrm{KCl}$ was used as the reference electrode.

\section{Results and Discussion}

\subsection{Surface characteristics of poly-TBO film coated by electropolymerization}

Figure 2 shows the cyclic voltammograms of the poly-TBO-modified electrodes subjected to electropolymerization in $1 \mathrm{M} \mathrm{HCl}$. The redox peak pairs of the poly-TBO film electrodes changed from about 0.23 and $0.38 \mathrm{~V}$ to about 0.35 and $0.40 \mathrm{~V}$ with increasing electropolymerization time. In particular, the redox peaks increased with increasing electropolymerization time, but the CV curves hardly changed after electropolymerization for 6 min. Thus, the polymerization process of TBO films on the Au electrodes is expected to change with the electropolymerization time and the modification of poly-TBO on the Au electrode by electropolymerization was sufficiently completed at around $6 \mathrm{~min}$.

Figure 3 shows the immobilization density of TBO on the Au electrodes calculated on the basis of the CV curves shown in Fig. 2. The immobilization density $D$ was calculated using

$$
D=\frac{Q}{2 F S},
$$

where $Q$ is the number of charges consumed by the redox reaction, $F$ is the Faraday constant, and $S$ is the reaction area of the electrode. $Q$ was calculated by integrating current with respect to time. In this case, two electrons took part in the redox reaction for each unit of poly-TBO [Fig. 1(b)]. ${ }^{(20)}$ The density increased linearly with increasing electropolymerization time up to 6 min, reaching $1.42 \pm 0.1 \mathrm{nmol} / \mathrm{cm}^{2}$, when it saturated.

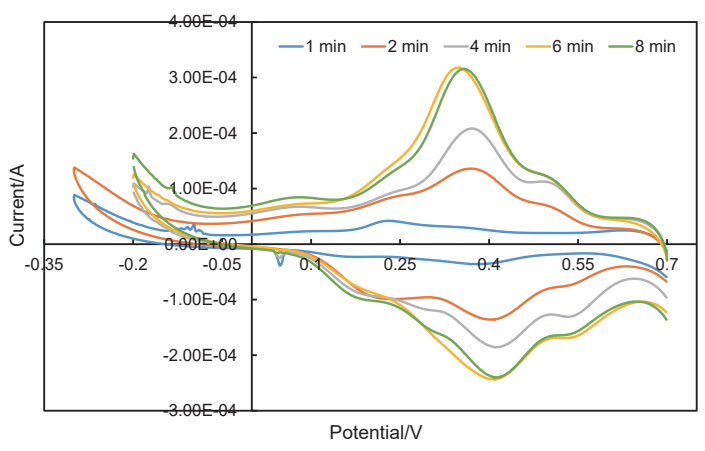

Fig. 2. (Color) Cyclic voltammograms of poly-TBO $\mathrm{Au}$ electrodes electropolymerized for 1 to $8 \mathrm{~min}$.

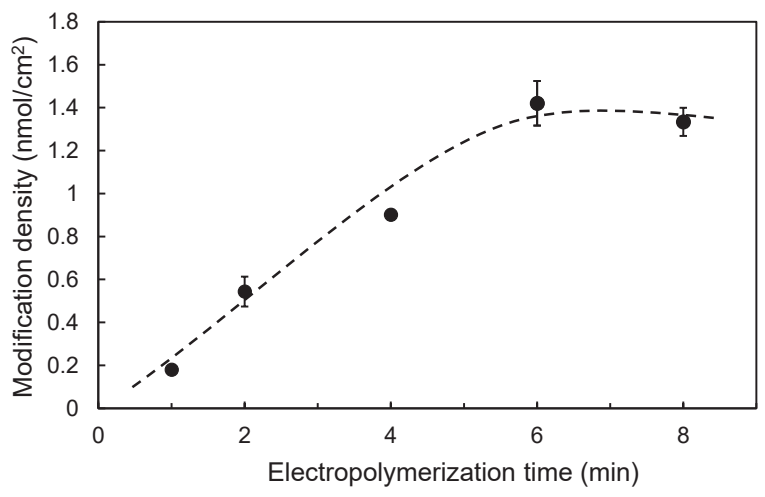

Fig. 3. Immobilization density of poly-TBO films electropolymerized for 1 to $8 \mathrm{~min}$. 
The surface morphology and thickness of the poly-TBO films on the Au electrodes were investigated by AFM as shown in Fig. 4. Figure 4(a) shows three-dimensional (3D) images obtained from AFM measurements of the Au electrode surfaces, which were untreated or modified by the electropolymerization of TBO for 1 or $6 \mathrm{~min}$. These images showed two regions of the glass substrate surface and the Au electrode surface treated or untreated by electropolymerization, although the TBO polymer was not modified near the edge between the glass substrate and the Au electrode. The roughness of the poly-TBO film gradually increased with the electropolymerization time, and the poly-TBO film developed a spikelike structure on the Au electrode. Corresponding to Fig. 4(a), the change in roughness during the polymerization process can also be seen in the cross-sectional profile shown in Fig. 4(b). In particular, the histograms of the height distribution for the unmodified and modified $\mathrm{Au}$ electrode surfaces shown in Fig. 4(c) revealed that the polymerization process produced different
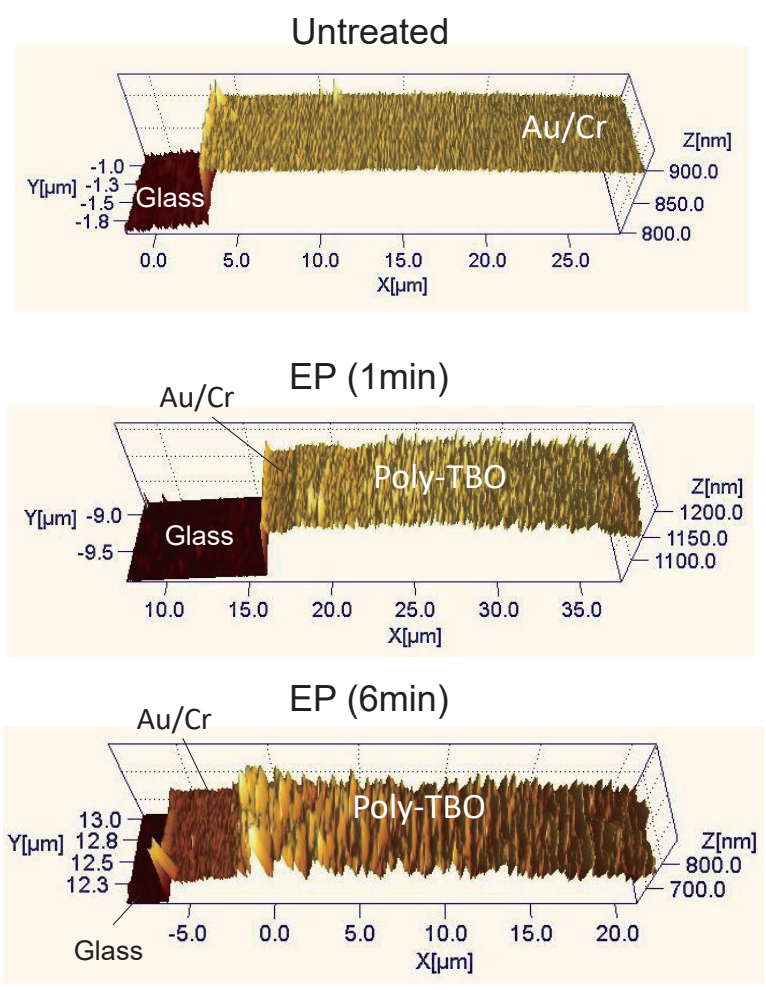

(a)

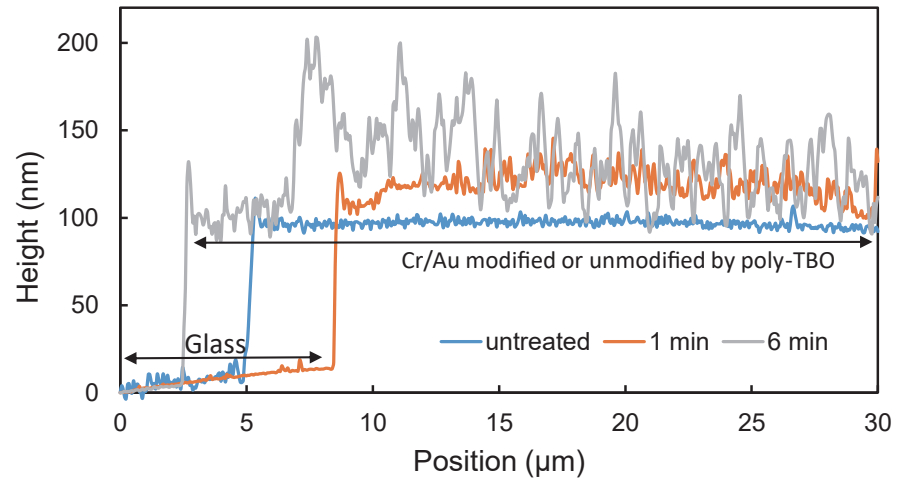

(b)

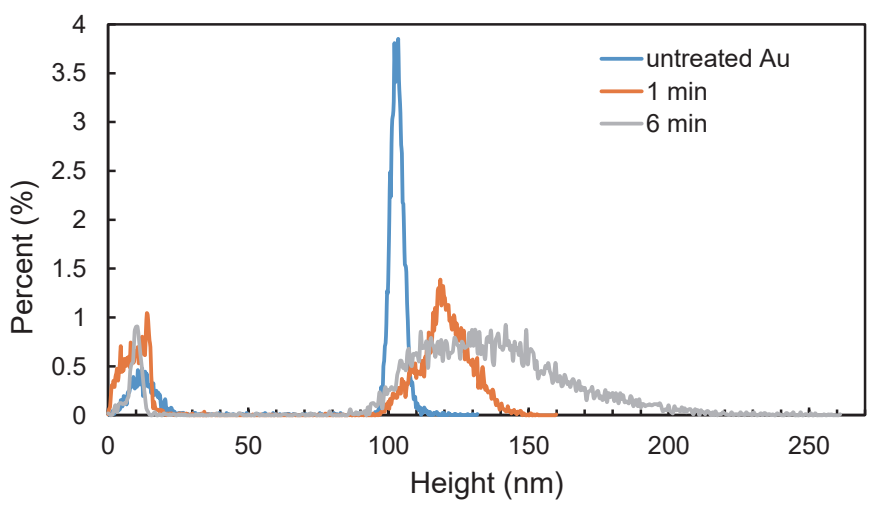

(c)

Fig. 4. (Color) Surface morphology and thickness of poly-TBO films electropolymerized for 1 and 6 min observed by AFM measurement. (a) 3D images of the $\mathrm{Au}$ electrode surfaces untreated and modified by the electropolymerization (EP) of TBO for 1 and $6 \mathrm{~min}$. The $x$-range is $30 \mu \mathrm{m}$ and the $y$-range is $1 \mu \mathrm{m}$. (b) Crosssectional profile on the center line of $y$-range for each electrode. The basis of the height was defined as the height at the start point of the glass surface in (b) and (c). These profiles showed the change in roughness during the polymerization process. (c) Histograms of height distribution of the unmodified Au electrode and the Au electrodes modified for 1 and 6 min shown in (a). Two peaks appeared for each Au electrode; the first peak (left) originated from the glass substrate surface without the $\mathrm{Au} / \mathrm{Cr}$ film and the second peak (right) originated from the $\mathrm{Au} / \mathrm{Cr}$ film surface. 
roughnesses. For the unmodified Au electrode, the peak frequency was very sharp; that is, the Au surface obtained by sputtering was flat. On the other hand, the height distributions for the $\mathrm{Au}$ electrodes covered with the poly-TBO film gradually became broad with increasing electropolymerization time. This means that the poly-TBO film treated for 1 min was relatively flat, but the film reacted for $6 \mathrm{~min}$ had a rough surface owing to the spikelike structure. Moreover, the height with the peak frequency increased with increasing electropolymerization time because the thickness of the poly-TBO film increased with the formation of the spikelike structure. The heights of the poly-TBO films coated by electropolymerization were about 10 to $20 \mathrm{~nm}$ after 1 min of treatment and about 30 to $60 \mathrm{~nm}$ after 6 min of treatment.

\subsection{Use of poly-TBO electrode as potentiometric biosensor}

The poly-TBO films coated by electropolymerization for 1 and 6 min were focused on to investigate the electrical properties of the modified electrodes; these films are hereafter referred to as the $1 \mathrm{~min}$ and $6 \mathrm{~min}$ poly-TBO films, respectively. Figure 5 shows the change in surface potential $\left(\Delta V_{\text {out }}\right)$ at the poly-TBO-coated $\mathrm{Au}$ electrode as a function of $\mathrm{pH}$. As shown in Fig. 5(a), both poly-TBO films showed $\mathrm{pH}$ responsivity within the $\mathrm{pH}$ range of 8.1 to 2.5 at
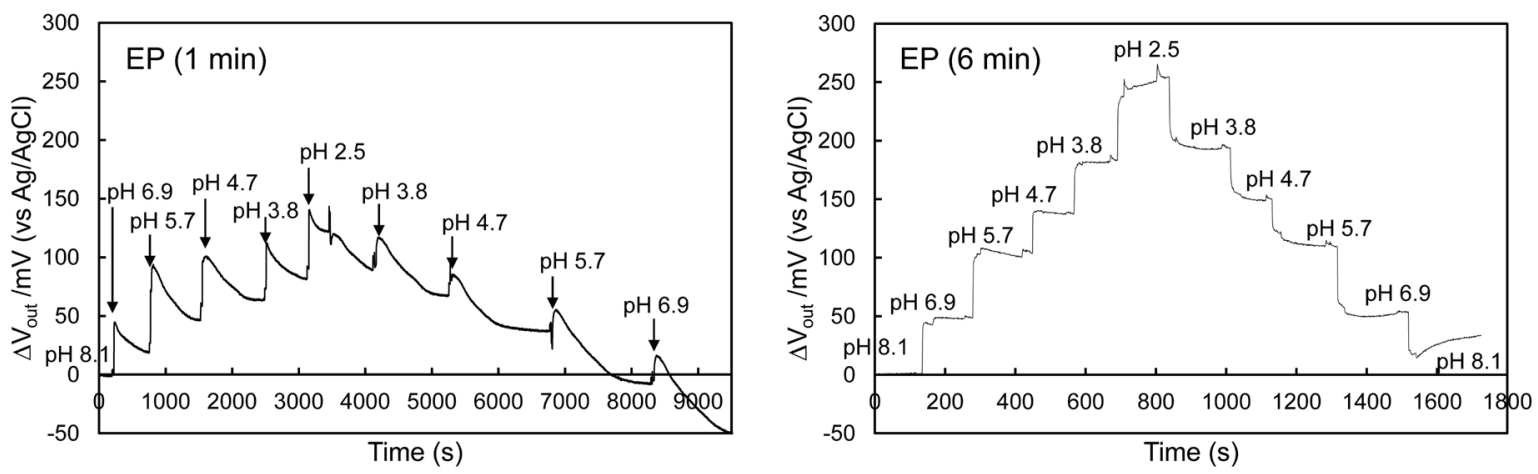

(a)

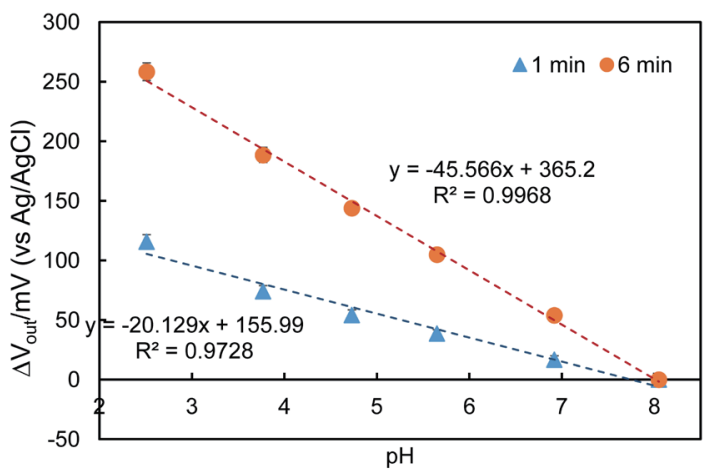

(b)

Fig. 5. (Color online) Change in surface potential $\left(\Delta V_{\text {out }}\right)$ at the poly-TBO-coated Au electrode as a function of $\mathrm{pH}$. (a) Real-time monitoring of $\Delta V_{\text {out }}$ for $\mathrm{pH}$ values between 8.1 and 2.5 using the poly-TBO-coated Au electrodes subjected to EP for 1 (left graph) and 6 (right graph) min. (b) Calibration curve of $\Delta V_{\text {out }}$ for different $\mathrm{pH}$ values on the basis of the real-time measurements shown in (a). 
intervals of about $1 \mathrm{pH}$ unit. However, a few differences were found between the 1 min and 6 min poly-TBO films: the potential drift was greater for the 1 min poly-TBO film than for the 6 min poly-TBO film; $\Delta V_{\text {out }}$ for the 6 min poly-TBO film showed greater variation with $\mathrm{pH}$ than that for the $1 \mathrm{~min}$ poly-TBO film; $\Delta V_{\text {out }}$ for the 6 min poly-TBO film almost recovered when $\mathrm{pH}$ was restored to its initial value of 8.1 in a stepwise manner, but such stable responses were not obtained for the 1 min poly-TBO film. By considering the electrical measurements in real time, $\mathrm{pH}$ sensitivity were evaluated to be $20.1 \mathrm{mV} / \mathrm{pH}$ for the 1 min poly-TBO film and 45.5 $\mathrm{mV} / \mathrm{pH}$ for the 6 min poly-TBO film as shown in Fig. 5(b). Thus, the 6 min poly-TBO film showed superior $\mathrm{pH}$ sensitivity to the $1 \mathrm{~min}$ poly-TBO film. This may be due to the difference in surface morphology originating from the different electropolymerization times. That is, the surface of the 6 min poly-TBO film had a spikelike structure, while that of the 1 min polyTBO film was relatively flat owing to the short polymerization time. As shown schematically in Fig. 6, the charge density at a spikelike film is assumed to be higher than that at the smooth film; therefore, $\Delta V_{\text {out }}$ for the spikelike film was larger than that for the smooth film. In fact, the monomer and dimer or oligomer of TBO include amino groups [Fig. 1(a)], ${ }^{(20)}$ which undergo an equilibrium reaction with hydrogen ions; that is, TBO exhibits $\mathrm{pH}$ responsivity, which is why the poly-TBO films showed $\mathrm{pH}$ responsivity. Additionally, the spikelike structure is considered to have contributed to the improved $\mathrm{pH}$ responsivity and sensitivity. Basically, the $\mathrm{pH}$ response of the oxide gate surface should be Nernstian when potentiometric sensors are used; ${ }^{(28,29)}$ the Nernstian response is calculated to be $59.1 \mathrm{mV} / \mathrm{pH}$ at $25^{\circ} \mathrm{C}$ using

$$
\begin{gathered}
E=E^{0}+\frac{2.30 R T}{n F} \log \left[H^{+}\right], \\
\Delta E=59.1 \cdot \Delta p H,
\end{gathered}
$$

where $E^{0}$ is the standard electrode potential, $R$ is the gas constant, $T$ is the absolute temperature, and $n$ is the valence of the ion $(n=1$ for $\Delta p H)$. In this study, a $\mathrm{pH}$ sensitivity of $45.5 \mathrm{mV} / \mathrm{pH}$ was obtained for the spikelike poly-TBO film, which is near the Nernstian response. Thus, a

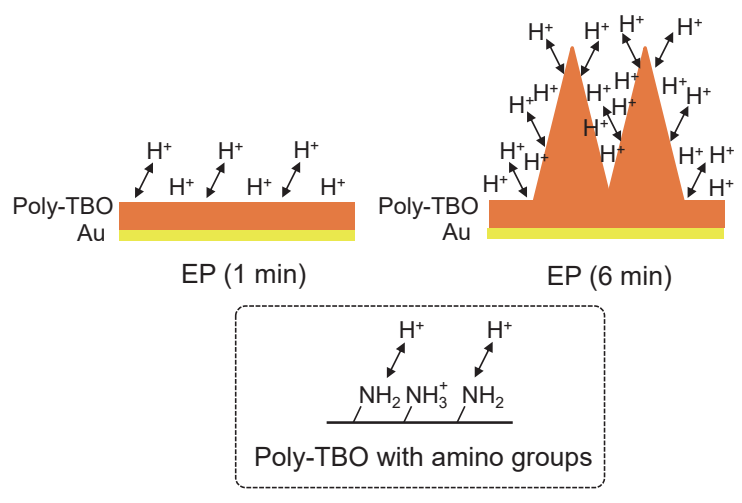

Fig. 6. (Color online) Schematic illustration of poly-TBO film surfaces obtained by EP for 1 and 6 min. 
platform based on a potentiometric biosensor with a poly-TBO film electrode can be used as a bioanalytical system to measure ionic behaviors that involve biomolecular recognition events, such as the TBO-sGAG interaction, as well as $\mathrm{pH}$ variations.

\section{Conclusions}

In this study, we investigated the fundamental properties of poly-TBO film electrodes for use as potentiometric biosensors for sGAG sensing. Negatively charged sGAG molecules are included in the ECM generated from chondrocytes and are used as an important indicator in the development of articular cartilage in the field of regenerative medicine because of their electrostatic binding to positively charged TBO molecules. We modified a poly-TBO film on a $\mathrm{Au}$ electrode by electropolymerization and investigated its electrical characteristics to evaluate its use as a potentiometric biosensor. The fundamental properties of poly-TBO films, such as immobilization density and thickness, were clarified as functions of the electropolymerization time by AFM. The poly-TBO film electrode with a spikelike surface that formed during electropolymerization showed good $\mathrm{pH}$ sensitivity $(45.5 \mathrm{mV} / \mathrm{pH})$, which was near the Nernstian response, indicating its suitability for use as a potentiometric sensor. This response is assumed to arise because the charge density at a spikelike film was higher than that at a smooth film. Thus, a platform based on a potentiometric biosensor with a poly-TBO film electrode can be used as a bioanalytical system to measure ionic behaviors that involve biomolecular recognition.

\section{Acknowledgments}

We would like to thank Professor S. Mizuno of Harvard Medical School for help and useful discussions.

\section{References}

1 E. B. Hunziker: Osteoarthr. Cartilage 7 (1999) 15.

2 M. Brittberg, A. Lindahl, A. Nilsson, C. Ohlsson, O. Isaksson, and L. Peterson: New, Engl, J. Med. 331 (1994) 889.

3 M. D. Buschmann, Y. A. Gluzband, A. J. Grodzinsky, J. H. Kimura, and E. B. Hunziker: J. Orthop. Res. 10 (1992) 745.

4 J. K. Mouw, N. D. Case, R. E. Guldberg, A. H. K. Plaas, and M. E. Levenston: Osteoarthr. Cartilage 13 (2005) 828.

5 J. Elisseeff, W. Mclntosh, K. Anseth, S. Riley, P. Ragan, and R. Langer: J. Biomed. Mater. Res. 51 (2000) 164.

6 A. M. Freyria, M. C. Ronziere, S. Roche, C. F. Rousseau, and D. Herbage: J. Cell. Biochem. 76 (1999) 84.

7 J. J. Jeffrey and G. R. Martin: Biochim. Biophys. Acta 121 (1966) 269.

8 A. G. Glark, A. L. Rohrbaugh, I. Otterness, and V. B. Kraus: Matrix Biol. 21 (2002) 175.

9 J. D. Kisiday, B.Kurz, M. A. DiMicco, and A. J. Grodzinsky: Tissue Eng. 11 (2005) 141.

10 X. Liu, J. Liu, N. Kang, L. Yan, Q. Wang, X. Fu, Y. Zhang, R. Xiao, and Y. Cao: Int. J. Mol. Sci. 15 (2014) 1525.

11 K. H. Chua, B. S. Aminuddin, N. H. Fuizina, and B. H. I. Ruszymah: Eur. Cells Mater. 9 (2005) 58.

12 P. C. Yaeger, T. L. Masi, J. L. de Oritz, F. Binette, R. Tubo, and J. M. McPherson: Exp. Cell. Res. 237 (1997) 318.

13 S. D. Waldman, C. G. Spiteri, M. D. Grynpas, R. M. Pillar, and R. A. Kandel: J. Orthop. Res. 21 (2003) 590.

14 B. D. Elder and K. A. Athanasiou: Tissue Eng. 15 (2009) 42. 
15 T. Ikenoue, M. C. Trindade, M. S. Lee, E. Y. Lin, D. J. Schurman, S. B. Goodman, and R. L. Smith: J. Orthop. Res. 21 (2003) 110.

16 S. Mizuno, T. Tateisi, T. Ushida, and J. Glowacki: J. Cell. Phys. 193 (2002) 319.

17 H. Satake, A. Saito, S. Mizuno, T. Kajisa, and T. Sakata: Jpn. J. Appl. Phys. 56 (2017) 04CM03.

18 R. W. Farndale, C. A. Sayers, and A. J. Barrett: Connect. Tissue Res. 9 (1982) 247.

19 R. Ogawa, S. Mizuno, G. F. Murphy, and D. P. Orgill: Tissue. Eng. Part A 15 (2009) 2937.

20 D. M. Zhou, J.-J. Sun, H.-Y. Chen, and H.-Q. Fang: Electrochim. Acta 43 (1998) 1803.

21 A. F. Diaz, K. K. Kanazawa, and G. P. Gardini: J. Chem. Soc., Chem. Commun. 0 (1979) 635.

22 G. Tourillon and F. Gamier: J. Electroanal. Chem. 135 (1982) 173.

23 I. Rubinstein: J. Electrochem. Soc. 130 (1983) 1506.

24 N. Oyama, T. Ohsaka, T. Hirokawa, and T. Suzuki: J. Chem. Soc., Chem. Commun. 0 (1987) 1133.

25 A. F. Diaz and J. A. Loganm: J. Electroanal. Chem. 111 (1980) 111.

26 I. Sekine, K. Kohara, T. Sugiyama, and M. Yuasa: J. Electrochem. Soc. 139 (1992) 3090.

27 K. Naoi, Y. Oura, Y. Iwamizu, and N. Oyama: J. Electrochem. Soc. 142 (1995) 354.

28 T. Sakata, K. Nishimura, Y. Miyazawa, A. Saito, H. Abe, and T. Kajisa: Anal. Chem. 89 (2017) 3901.

29 H. Yang, M. Honda, A. Saito, T. Kajisa, Y. Yanase, and T. Sakata: Anal. Chem. 89 (2017) 12918. 